\title{
Formación de profesores de matemáticas: los caminos del profesionalismo docente en la historia de la educación brasileña
}

\author{
Math teacher training: the paths of teacher \\ professionalism in the history of Brazilian education \\ Formação de professores de matemática: os caminhos da \\ profissionalidade docente na história da educação brasileira
}

IRACEMA CAMPOS CUSATIDa

ANTONIO CARRILlo AVELAR (iDb

\section{Resumen}

Este artículo presenta aspectos relacionados con la discusión actual sobre al rol de la práctica y profesionalización de la docencia para comprender los factores responsables de las vulnerabilidades observadas en la enseñanza de las matemáticas en Brasil que se explican por discontinuidades en la formación (inicial y continuada). Existe una brecha entre formación universitaria de profesores de matemáticas y práctica docente en la escuela básica, además de otras dicotomías que rodean el debate. El proceso de elaboración y aprobación del BNCC impuso un currículo homogéneo en una lógica centralizadora disociada de demandas formativas y de realidades locales del complejo y desigual sistema educativo brasileño. Las transformaciones sociales, políticas, económicas y culturales del mundo contemporáneo afectan los sistemas educativos. La investigación bibliográfica y documental realizada sobre la formación del profesorado (PEREIRA, 2012) concluyó en la necesidad de cualificación, formación, rescate de la profesionalidad y políticas de profesionalización ante la devaluación y precariedad de la formación inicial docente. Avalos (2021), Ponte (2005) y Gatti, Barretto \& André (2011) muestran que los planes de estudio de las carreras de matemáticas no articulan conocimientos técnico-científicos y pedagógicos, desvelando la dicotomía

\footnotetext{
a Universidade de Pernambuco (UPE), Petrolina, PE, Brasil. Doutora em Educação, e-mail: iracema.cusati@gmail.com

b Universidade Pedagógica Nacional (UPN), Cidade do México, DF, México. Doutor em Ciências Antropológicas, e-mail: antoniocarrillobr@hotmail.com
} 
entre matemáticas académica y escolar. La forma reduccionista de integración entre teoría y práctica niega la necesidad de una sólida formación teórica e interdisciplinar, difundiendo una perspectiva del conocimiento restringido al saber hacer, marcado por ausencia de procesos reflexivos en la formación docente. Los aportes presentados orientan la movilización de conocimientos necesarios para la profesionalización de la docencia, subvencionando la construcción de la identidad profesional de los docentes.

Palabras clave: Formación de profesores de matemáticas. Profesionalización docente. Formación didáctica.

\section{Abstract}

This article presents the aspects related to the current discussion on the role practical and of the professionalization of the teaching in order to understand the factors responsible for the vulnerabilities observed in the teaching of mathematics in Brazil that are, large extent, explained by the discontinuities in training (initial and lifelong learning) of teachers. There is a gap between university education of mathematics teachers and teaching practice in basic school, in addition to several dichotomies that surround the debate. The process of elaboration and approval of the BNCC imposed a homogeneous curriculum in a centralizing logic dissociated from the training demands and the local realities of the complex and unequal Brazilian educational system. The social, political, economic and cultural transformations of the contemporary world affect educational systems. The bibliographic and documentary research carried out on teacher education (PEREIRA, 2012) concluded by the need for qualification, training, rescue of professionalism and policies for the professionalization of teachers in view of the devaluation and precariousness of initial teacher training. Avalos (2021), Ponte (2005) and Gatti, Barretto \& André (2011) show that the mathematics degree curricula do not articulate technical-scientific and pedagogical knowledge, unveiling the dichotomy between academic and school mathematics. The reductionist form of integration between theory and practice denies the need for solid theoretical and interdisciplinary training, disseminating a perspective of knowledge restricted to know-how, marked by the absence of reflective processes in teacher training. The contributions presented provide guidance on the mobilization of knowledge necessary for the professionalization of teaching, subsidizing the construction of the teachers' professional identity.

Keywords: Mathematics teacher training. Teaching professionalization. Didactic training.

\section{Resumo}

Este artigo apresenta os principais aspectos relacionados a atual discussão sobre o papel da prática e da profissionalização da docência no Brasil com o objetivo de entender os fatores responsáveis pelas fragilidades observadas no ensino da matemática que são, em grande medida, explicados pelas descontinuidades na formação (inicial e contínua) de professores. Há distanciamento entre formação universitária de professores de 
matemática e prática docente na escola básica, além de diversas dicotomias que cercam o debate. O processo de elaboração e aprovação da BNCC impôs uma homogeneização curricular numa lógica centralizadora dissociada das demandas formativas e das realidades locais do complexo e desigual sistema educacional brasileiro. As transformações sociais, políticas, econômicas e culturais do mundo contemporâneo afetam os sistemas educacionais. A pesquisa bibliográfica e de cunho documental desenvolvida sobre professoralidade (PEREIRA, 2012) concluiu pela necessidade de qualificação, formação, resgate da profissionalidade e das políticas de profissionalização de docentes tendo em vista a desvalorização e a precarização da formação inicial de professores. Avalos (2021), Ponte (2005) e Gatti, Barretto \& André (2011) mostram que os currículos de licenciatura em matemática não articulam saberes técnico-científicos e pedagógicos desvelando a dicotomia entre matemática acadêmica e escolar. A forma reducionista de integração entre teoria e prática nega a necessidade de uma sólida formação teórica e interdisciplinar, disseminando uma perspectiva de conhecimento restrita ao saber fazer, marcada pela ausência dos processos reflexivos na formação docente. As contribuições apresentadas orientam sobre a mobilização de saberes necessários à profissionalização da docência subsidiando a construção da identidade profissional dos professores.

Palavras-chave: Formação de Professores de matemática. Profissionalização docente. Formação didática.

"Para ver de verdad hay que comparar lo que se ve con lo que se ha visto"

(PAZ, Octavio. Repaso en forma de preámbulo, In "Los Privilegios de la Vista". Fondo de Cultura Economica: México, 1987. p. 16-17).

\section{Introducción}

Este artículo presenta los principales aspectos relacionados con la discusión actual sobre la profesionalización de la docencia en Brasil con el fin de comprender los factores responsables de los problemas observados en la enseñanza y el aprendizaje de las matemáticas que se explican, en gran medida, por las discontinuidades en la formación (inicial y continua) de los profesores. Para identificar los contextos de constitución de la profesionalidad de los docentes de matemáticas, se revisa la investigación que estudió la trayectoria formativa de estos sujetos para comprender cómo fueron construyendo el ethos profesional (forma de ser) y también para identificar, en sus trayectorias formativas, indicadores basados en un soporte teórico-práctico que nos permitió superar la encrucijada de este camino, salpicado de obstáculos e incertidumbres inherentes a la docencia, pero lleno de logros. 
Pimenta y Gonçalves (1992), al desarrollar un profundo estudio sobre la formación de docentes en Brasil entre 1930 y 1988, destacaron las profundas transformaciones políticas, económicas y sociales vividas en el país, desde 1930, y la creciente demanda de educación de la población. trabajador, que organizó y exigió escuelas gratuitas por ser "una condición de acceso al mercado laboral y, por tanto, de supervivencia” (PIMENTA y GONÇALVES, 1992, p. 98).

En las primeras décadas del siglo XXI germinan las investigaciones y discusiones sobre la profesionalidad docente, tema que expresa el carácter profesional de la docencia como profesión en acción y que he asumido, desde una perspectiva procedimental, el acto de enseñar como inherente a la función docente. Investigadores como Ponte (2005), Mizukami (2005-2006), Nóvoa (2009), Cunha (2010), Gatti et. al. (2011; 2019), Pereira (2012), Zeichner (2013) entre otros, nos ayudan a comprender los contornos de este debate en la educación actual.

Además de la formación prescrita, y teniendo como referencia el momento pandémico del coronavirus (Covid-19), que incentiva a los educadores a reinventar sus prácticas utilizando estrategias para la continuidad del proceso de aprendizaje presencial de los estudiantes, los impactos serán considerables y, aun así, no completamente dimensionado sobre la sociedad. Es un hecho inédito en la historia, dado que en el pasado se desarrollaron epidemias similares en un escenario de mucha menos interrelación entre personas, integración entre países, división del trabajo y densidad de población.

Muestra una crisis sin precedentes en el sistema educativo brasileño, que se extiende a las relaciones sociales, la política, la economía, la medicina, entre muchas otras áreas del conocimiento. En educación, las estrategias y soluciones que se adopten en el mundo para este momento difícil, por efímero que sea, traerán lecciones importantes que pasarán por la formación de los docentes, entornos de aprendizaje especialmente involucrando tecnologías digitales y políticas educativas que, cada vez más, deberán ser pensadas con responsabilidad. sociales y solidarios, factores que deben ser observados y considerados por los gestores educativos, los profesionales de la educación y las comunidades escolares.

Por ello, recabando datos y experiencias de diferentes partes del mundo respecto al cierre de escuelas para contener la propagación del coronavirus y mitigar los efectos de la pandemia en la educación, se vislumbran las consecuencias de esta 
acción que permean la vida escolar. Por lo tanto, es necesario cuestionar aún más la formación de los profesores de matemáticas que laboran y los que trabajarán en educación en el futuro, ante las adversidades, a partir de 2020. Se presume que todo profesional tiene allá de formación específica en profundidad en su área además de una competencia técnica y compromiso ético. Particularmente, en la formación del profesorado, estos elementos son fundamentales y, en una valoración sistémica del alcance de sus prácticas, se asume que todo alumno tiene derecho a aprender y si el alumno no aprendió, uno de los factores a considerar puede ser el modelo de formación del profesorado que no dio cuenta de su alcance.

Se demandan posiciones analíticas, críticas y técnicas de la comunidad docente para pensar propuestas que las redes educativas puedan implementar con el objetivo de promover el aprendizaje y el tiempo pedagógico de los estudiantes de manera equitativa pospandémica a fin de mitigar los riesgos asociados a la evasión, los índices de repetición o incluso cierre de escuelas según contextos sociales.

La formación, además de apoyar al profesorado en la evolución de sus carreras y en la resolución de los problemas acuciantes de la práctica profesional, necesita cada vez más orientarse hacia proyectos escolares, verdaderamente orientados a las necesidades de los estudiantes.

Hay una brecha entre la formación del profesorado universitario y la práctica del aula en la escuela básica, entre otras dicotomías que rodean el debate. La elaboración y aprobación del BNCC resulta de un proceso que impuso un currículo homogéneo en una lógica centralizadora que se disocia de las demandas formativas y de las realidades locales en el complejo y desigual sistema educativo brasileño. ¿Sería posible hacer, en las universidades, una reflexión sobre la formación inicial de los estudiantes de pregrado, contribuyendo a la formación de profesionales conscientes de la realidad educativa en la que trabajarán? A este cuestionamiento se suman otros específicos: ¿Está o está preparado este profesor de matemáticas para la realidad en la que actuará? En relación al docente recién egresado, ¿Su graduación le permitió un repertorio para la intervención que realizará? Y al docente, más experimentado, ¿la formación continuada le puso en contacto con posibilidades de demandas similares a las que le han pedido que asuma? Estas interrogantes surgieron ante las frecuentes incertidumbres en este momento pandémico, que ponen en jaque a la Educación y al 
rol del docente, al orientar la discusión hacia la formación y el desarrollo profesional de los docentes, en particular las matemáticas.

En este sentido, el tema en cuestión será el objetivo de este estudio, bibliográfico y documental, basado en una selección de bibliografía, elegida por la legitimidad académica, que no representa el estado del arte, pero que sobresale en amplitud y trascendencia para las reflexiones desarrolladas.

Las transformaciones sociales, políticas, económicas y culturales del mundo contemporáneo afectan los sistemas educativos y la producción historiográfica sobre el tema he propiciado un debate especializado para la valorización de la información proveniente de fuentes.

El aporte teórico enumerado en este texto incentiva la reflexión para comprender y explicar los factores responsables de las discontinuidades en la formación (inicial y continua) de los docentes e identificar los contextos de constitución de la profesionalidad de los docentes de matemáticas.

\section{Breve historia de la formación de profesores de matemáticas en Brasil}

La atención prestada a la formación para la transformación social, condición sine qua non, no es nueva. En la década de 1930, con las reformas llevadas a cabo por Anísio Teixeira, este sesgo ya se estaba discutiendo en los cursos iniciales de formación docente (ZEICHNER, 2008).

Los firmantes del Manifiesto de los Pioneros de la Nueva Educación de 1932 sobresalieron en la resolución de la situación de la educación pública en Brasil, considerando que el progreso económico y social del país estaba intrínsecamente ligado a las condiciones de falta de cultura propia y, sobre todo, de planes educativos sistemáticos. y eficaz. Inconformes con la organización educativa actual, estos pioneros exigieron que la enseñanza unificada y su laicidad sea gratuita y obligatoria.

Este sistema educativo también demandaba docentes con formación unificada, de carácter pedagógico (TEIXEIRA, 1996). El Manifiesto fue un documento político que abordó los problemas relacionados con la formación docente y la inexorable nueva actitud del educador para trabajar en la nueva escuela. 
Anísio Teixeira, pionero del Manifiesto de 1932 y destacado defensor de la educación brasileña, trajo grandes aportes a los problemas educativos del país cuyas matrices fueron influenciadas por el educador John Dewey, creador del nuevo movimiento escolar.

Entre las colaboraciones de Anísio Teixeira para el sistema educativo brasileño se encuentra la creación de la Universidad del Distrito Federal (Río de Janeiro) con sus cinco escuelas: la Facultad de Filosofía y Letras, la Facultad de Ciencias, Economía Política y Derecho, la Escuela de Educación y el Instituto de las Artes. Estas escuelas demarcaron las primeras experiencias en Brasil que tenían como objetivo la formación de docentes de nivel superior para trabajar en educación básica, materializando la preocupación de Anísio Teixeira por elevar el nivel de la profesión docente.

Al fin de la década de 1950, los profesores de matemáticas tenían antecedentes diversos y solo una minoría tenía un título en matemáticas o física. Debido a la falta de estudiantes de pregrado, muchos profesores de matemáticas eran ingenieros, educadores y personas sin formación regular en la zona. Este marco contribuyó a que, en general, los docentes se limitaran a repetir en el aula lo prescrito en los libros de texto o determinado en planes descontextualizados (AVALOS, 2021). Este fue el modelo de enseñanza de las matemáticas establecido y aplicado en las escuelas. Desde el punto de vista pedagógico, transmitía la idea de que "bastaba saber matemáticas para enseñar", desde que se tuvieran en cuenta algunos principios didácticos generales. Esta creencia, en la que el dominio del conocimiento matemático constituye una condición necesaria y suficiente para garantizar su transmisión, fue uno de los obstáculos para el éxito de la reforma de la enseñanza de las matemáticas denominada Movimiento de las Matemáticas Moderna (MMM). Si la primera condición es incuestionable, no se garantiza la misma en la segunda.

La euforia sobre las posibilidades del movimiento, cuyo foco era la reestructuración de las matemáticas como ciencia no fue suficiente para sensibilizar a los docentes sobre la importancia de los cambios propuestos y la enseñanza continuó replicando el programa prescrito. A medida que el carácter del contenido se exacerbó a partir de este movimiento, las cuestiones de orden metodológica fueron relegadas a un segundo plan, subyugadas a la naturaleza del contenido como conocimiento lógicamente estructurado. 
Considerando el proceso de conocimiento matemático como estático, formalizado y listo, en esta concepción, las matemáticas estaban fuera de contexto. La didáctica matemática fue impuesta por una serie de técnicas y procedimientos y el docente como mero usuario de las mismas. En esta etapa, se evidenció el alto grado de formalización de las matemáticas en la escuela primaria y secundaria, perspectiva que sirvió a una minoría de estudiantes que estudiaron Licenciatura en Matemáticas. Sin embargo, alejada de la mayoría de los estudiantes que lo usaban como herramienta de trabajo. La fase de sensibilización para el Movimiento era accesible para los profesores de matemáticas, pero ni siquiera garantizaba un programa que incluyera áreas afines y que buscara la integración con ellas. El Parecer No. 295, aprobado el 14 de noviembre de 1962, instituyó en Brasil un plan de estudios mínimo para los grados de Matemáticas.

En la década de 1970, los procesos de formación y profesionalización docente en Brasil se caracterizaron por la desarticulación de los problemas que enfrentan los profesionales de la educación en su vida diaria.

Con la implementación de la reforma curricular a través de la Ley 5692/71, se introdujo toda la programación de las matemáticas tanto en la educación primaria como en la secundaria, aunque su carácter histórico y político quedó excluido del proceso educativo.

La década de 1970 estuvo marcada por dos fases distintas, una de entusiasmo con la consolidación del uso de las matemáticas en las escuelas, acompañada de otra fase de análisis críticas y debates sobre su comprensión y uso. Sin duda, el Movimiento de las Matemáticas Modernas reforzó aún más la percepción lineal de la transmisión del conocimiento (docente-alumno), ya que en la forma de organización del trabajo escolar predominaba el marco arbitrario de prerrequisitos y la enseñanza desmotivada, trayendo consecuencias relevantes para la educación. estudiante que no aprendió ni la nueva ni la vieja matemáticas (MOTEJUNAS, 1989).

A partir de la década de 1980, el número de licenciados en matemáticas aumentó debido a que, durante la expansión de la educación superior, uno de los cursos más autorizados para trabajar fue matemáticas porque no necesitaba laboratorios ni salas especiales. El movimiento se impuso, con sus ventajas y desventajas, sin embargo, la formación de los profesores de matemáticas fue empeorando considerablemente, a pesar de que formaron más profesores (MOTEJUNAS, 1989). 
El resultado de las matemáticas en transición se refleja y refleja en la formación y actuación de los docentes, en el reconocimiento de un conocimiento pedagógico de las matemáticas que se ha ido consolidando como un área de investigación y aportando numerosos aportes metodológicos.

Se cuestionaron algunas marcas de la implantación del Movimiento de las Matemáticas Moderna, como el trabajo con conjuntos, el predominio de temas algebraicos sobre los geométricos, el tratamiento de la geometría como tema ilustrativo de conjuntos o álgebra, el exceso de lenguaje simbólico y la crítica cobró fuerza a lo largo de la década de los 80 al final del siglo pasado.

Según Bicudo (1987), el docente formado en matemáticas o áreas de conocimiento afines, está muy preocupado por las matemáticas utilizadas como un cuerpo de conocimientos organizado de forma lógica y explicitado en un lenguaje específico. Sin embargo, ante situaciones específicas de enseñanza, ese maestro se da cuenta, por una serie de factores, de que el conocimiento científico no es suficiente para garantizarle el éxito en la docencia.

La práctica dialéctica de reflexión-acción-reflexión provoca sensaciones variadas en el docente, ya que constantemente se enfrenta a grandes cambios en cuanto a su actitud ante la enseñanza-aprendizaje y al alumno, así como respecto al cuerpo de conocimientos matemáticos a construir.

Las matemáticas, como práctica pedagógica, deben conducir a una visión global entre la realidad y la sociedad. Tiene sus códigos y su lenguaje, tiene un sistema de comunicación y representación de la realidad construido paulatinamente a lo largo de la historia. Para comprenderlo e interpretarlo, es necesario comprender la organización y lógica de su lenguaje, así como su significado construido históricamente.

En consecuencia, a medida que el alumno no llega a comprender los conceptos matemáticos, acumula cada vez más dudas, incertidumbres y miedos, mientras se esfuerza por apropiarse de nuevos contenidos basados en información vista previamente, pero que no se transformó en conocimiento.

No es nuevo que la educación pública brasileña, en cualquier nivel o área curricular, enfrente serios problemas, entre ellos la necesidad de revertir la tasa de deserción, retención y baja calidad educativa. En particular, la enseñanza de las 
matemáticas no presenta diferentes aspectos y se añaden cuestiones relacionadas con el desinterés de los estudiantes por los contenidos, la dificultad para comprender los conceptos abordados, la desconexión de la práctica desarrollada por el docente en el aula con situaciones concretas etc. (MOTEJUNAS, 1989).

En realidad, existe una situación crítica en la educación en general, con causas conocidas, pero que trascienden el ámbito particular de las matemáticas. Sin embargo, esta situación se refleja, con gran intensidad, en esta disciplina, en parte porque es una de las pocas que aún se enseñan de forma continua, jerárquica y secuenciada. Tu enseñanza solo será efectiva cuando todos entiendan que, además de alterar los contenidos, que ya se ha hecho parcialmente con la introducción de las matemáticas llamadas Moderna, también es necesario cambiar las metodologías, desde las estrategias para abordar las asignaturas hasta los criterios de evaluación.

Afortunadamente, las experiencias exitosas han sido desarrolladas por académicos y podemos mencionar el trabajo de Rangel; Giraldo e Maculan (2013) que desarrollan estudios colectivos en escenarios donde los docentes comparten sus conocimientos sobre temas matemáticos que se enseñan en la escuela primaria.

\subsection{Formación del profesorado: 'gap' entre la universidad y la escuela}

Una de las críticas que se suele hacer a la formación docente es la distancia entre la formación inicial y la práctica docente (LIMA y PIMENTA, 2004). Los docentes se quejan de que la formación académica no es suficiente para asumir la práctica pedagógica en un aula porque, frente a un grupo de alumnos, no se sienten preparados o no ven correspondencia entre lo estudiado y la práctica (NÓVOA, 2009; KLEIN, 2009). Como resultado de este proceso, estos profesionales no son capaces de actuar como transformadores del entorno en el que trabajan (ZEICHNER, 2008; 2013).

Esta crítica la evidencia Diniz-Pereira (2011), cuando muestra que en la redacción de la Ley de Lineamientos y Bases de la Educación Nacional (Ley n ${ }^{\circ}$ 9.394/96), los artículos 61 y 65 destacan la necesidad de una formación que acerque la práctica. de la teoría. Concomitantemente, es evidente que existe una preocupación en los cursos de formación docente por tener asignaturas que discutan la necesidad 
de que el docente sea un transformador social, pero no siempre brindan la oportunidad para la experiencia. En este sentido, el estudiante que vive esta contradicción en su educación, ante la enseñanza diaria, no está efectivamente preparado para ser un agente de una educación que transforme la realidad (ZEICHNER, 2008; 2013).

Una de las razones de estas disociaciones parece estar en la tensión entre la academia y la comunidad. Mientras la academia pretenda poder hacer los análisis sobre una educación enfocada en la justicia social, desconociendo las interpretaciones y aportes de la comunidad, habrá un modelo de enseñanza ineficaz (ZEICHNER, 2008; 2013).

La forma reduccionista de integración entre teoría y práctica niega la necesidad de una sólida formación teórica e interdisciplinar, difundiendo una perspectiva del conocimiento restringida al saber hacer, marcada por la ausencia de procesos reflexivos en la formación docente. Sin embargo, es urgente que para asegurar el aprendizaje docente y el desarrollo profesional de los docentes, exista una articulación de la formación inicial con la formación en servicio en una perspectiva de aprendizaje permanente; especial atención a los primeros años de práctica profesional y la inserción de jóvenes docentes en las escuelas; valorar el profesorado reflexivo y la formación del profesorado basada en la investigación; la importancia de las culturas colaborativas, el trabajo en equipo, el seguimiento, la supervisión y la evaluación por parte de los profesores; entre otros innumerables aspectos (NÓVOA, 2009).

En el contexto de la curricularización de la diversidad cultural presente en el escenario contemporáneo, le corresponde al docente comprender la complejidad que le es peculiar, pero también las vívidas relaciones históricas y prácticas que constituyen este contexto. Frente a la diversidad cultural, económica, política, histórica y subjetiva de cada alumno y a las concepciones arraigadas de los formadores, el desafío es desvelar estas diversidades al mundo como procesos conscientes y constituyentes del ser, que deben ser considerados cuestionando los propios lentes a través de la que contemplan y forjan la realidad.

En cuanto a la relevancia social de la formación de profesores de matemáticas, es inevitable rescatar a Bishop (1991) para quien el currículo debe enfatizar las matemáticas como un fenómeno cultural que puede ser una rica fuente de explicaciones en un camino de buen aprendizaje. Esta característica debe ser incorporada en los 
planes de estudio de la escuela básica y la universidad, instituciones que brinden acceso a conocimientos eruditos, sistematizados y, en consecuencia, garanticen la apropiación de los conocimientos históricamente producidos y la expresión elaborada de los contenidos de la cultura popular. El desafío que se debe enfrentar en la formación y actuación docente se refiere al énfasis que se les ha dado a las aplicaciones prácticas de las matemáticas en detrimento de las investigaciones matemáticas.

En sus trabajos, Bishop (1991) destaca que un currículo debe tener una concepción relativamente amplia y elemental, en lugar de ser limitado y detallado. El currículum escolar, que autoriza o no permite, legitima o deslegitima, incluye o excluye, no debe concebirse fuera de la interacción dialógica entre escuela y vida. En el campo de la formación docente, debe incorporar temas actuales y desafiantes para la práctica pedagógica. En el contexto escolar, exige una construcción colectiva para enfrentar los desafíos que presenta la diversidad cultural, es decir, enfocarse en la diferencia e interacción entre culturas permitiendo la construcción de la identidad de los diversos grupos que componen la sociedad brasileña.

Las prácticas curriculares, sus relaciones, sus sentidos y significados, así como las nuevas condiciones de formación y actuación docente, plantean desafíos para transformar la acción educativa. En el escenario actual, la temática de la formación docente ha despertado el interés de investigadores del área de la educación preocupados por la formación y práctica pedagógica de los docentes. Estos estudios tienen, en resumen, el propósito de describir la importancia de la práctica de este profesional en la escuela y en la sociedad y, en muchos, es recurrente una ilusoria y masiva posibilidad de éxito académico de los estudiantes. En esta perspectiva, es posible afirmar que la práctica pedagógica, al ser compleja, requiere del docente una reflexión diaria que se materialice en acciones. Por lo tanto, corresponde a la formación asegurar el enfoque en la docencia, en la investigación del conocimiento, en la valoración del conocimiento, en la autonomía pedagógica, en el uso de instrumentos didácticos y en los nuevos instrumentos tecnológicos, además de la apuesta por una educación democrática.

La reflexión sobre el compromiso social como cuestión de educar para la justicia social fue defendida por Zeichner (2008). Ser un educador con perspectiva de justicia social significa estar atento a la realidad del alumno y la comunidad en la que 
se inserta la escuela, estar comprometido con su función, buscando promover el cambio social en el entorno en el que se desenvuelve, entendiendo que existen múltiples visiones del mundo. $y$, finalmente, saber lidiar con la diversidad de sus alumnos y compañeros.

Ante tal escenario, una posible estrategia para la superación de estos problemas es la creación de espacios de formación híbridos, promoviendo un diálogo entre los docentes que trabajan en la educación básica y la educación superior para una mejor formación de los estudiantes de pregrado (ZEICHNER, 2010). En Brasil, la Resolución CNE/CP 02/2015 trajo grandes cambios en la dinámica de las pasantías curriculares de formación docente al señalar que es necesario ampliar la carga de trabajo relacionada con los cursos de pregrado.

Según Zeichner (2010; 2013), el diálogo acerca a las universidades a la realidad de las escuelas y promueve una relación más democrática entre las partes, ya que valora el conocimiento producido en ambas, contribuyendo a una mayor articulación entre la práctica y la teoría, permitiendo incluso la incorporación de nuevos elementos al currículo de formación docente adoptado en la academia.

Para que se capacite a los nuevos docentes de modo más democrático, Zeichner (2013) afirma que es necesario valorar el conocimiento comunitario en los lugares donde estos docentes trabajarán.

\section{Formación docente inicial y continua: viejos problemas en un nuevo entorno educativo}

En un campo que persiste en la diversidad y pluralidad, tanto conceptual como metodológica, los estudios sobre desarrollo profesional docente y profesionalización docente, según Mizukami (2004, p. 34), "si bien sirven como marcos explicativos," se requiere el desarrollo de investigaciones que muestran sus limitaciones y sus avances en relación a la producción de la zona “[...] y que algún día los superarán en sus puntos más vulnerables y reduccionistas y que aún no pueden dar cuenta de explicar por sí mismos los procesos de aprendizaje profesional enseñando".

La formación inicial del profesorado tiene como objetivo formar profesionales con competencia para el ejercicio de la profesión, pero estimar las habilidades que debe tener el docente en los ámbitos cognitivo, afectivo, ético y social en una 
profesión inminentemente relacional no es una tarea sencilla. Sin duda, los objetivos de la formación parten de la necesidad de que el docente se convierta en un miembro activo de su escuela y de su comunidad profesional.

Cuando consideramos que la formación inicial es parte de una formación continua de los docentes, como sugiere Pimenta (2000), no se puede ignorar la construcción del currículo en este proceso y los desafíos de la vida cotidiana en el aula requieren una formación continua por parte del docente.

Una política de formación continua implica acciones efectivas con el fin de mejorar la calidad de la docencia, las condiciones laborales y contribuir también a la evolución funcional de los docentes.

Como consecuencia de que hoy toda formación es formación inicial y que el docente debe asimilar la idea de formación continua a lo largo de los años de docencia, los planes de estudio de los cursos de formación docente deberían incorporar esta perspectiva.

La formación continua es un requisito de la actividad profesional del profesorado y no solo una compensación de las debilidades encontradas en la formación inicial. Se trata de aprendizaje que va más allá de la simple aplicación de lo estudiado, en una confluencia entre formación y profesionalización, en busca del desarrollo docente, respetando los valores, la cultura y los conocimientos en un aprendizaje profesional constante de la enseñanza.

Para Candau (1996), la formación continua de los docentes se configura como un aspecto relevante para la implementación de una propuesta que propone una renovación de las prácticas pedagógicas y las escuelas. Gatti y Barreto (2009) agregan que la renovación y mejora de la calidad en el desempeño de los docentes y en el aprendizaje implica considerar la formación, las carreras y las perspectivas profesionales de los docentes.

La importancia que se deposita a la formación continua de los docentes busca satisfacer las necesidades de los docentes que se encuentran trabajando en las escuelas y hacer frente a los nuevos y rápidos cambios en el ámbito de la sociedad, que repercuten en la escuela. Esta premisa en relación a la educación continua necesita considerar la cultura del profesorado y la cultura escolar en lugar de crear y fortalecer mecanismos de control, intensificación de la proletarización y precariedad del trabajo docente. 
La percepción de esta brecha en la formación docente refuerza la comprensión de la importancia de la educación continua o en servicio en un contexto cultural y temporal.

Existe un consenso entre teóricos e investigadores del área de formación docente sobre la imperiosa necesidad de la formación continua y la reconfiguración de la práctica docente a partir de un proceso continuo de acción — reflexión acción que parte de las propias experiencias y registros de los docentes.

La posibilidad de desarrollar una conciencia profesional, comprometida con prácticas reflexivas y críticas, comprometida en un trabajo colectivo en torno a la construcción de un proyecto político-pedagógico de una escuela de calidad para las clases populares, como señala Freire (2001), es un desafío planteado tanto a nivel individual como en el ámbito del trabajo colectivo. Este desafío se hace explícito cuando afirma que el objetivo principal de la escuela es la educación del alumno para ejercer su ciudadanía y no solo para formar un profesional integrado en el mercado laboral. Para ello, el docente debe ser un estimulador del aprendizaje y también actuar como interviniente durante el proceso de aprendizaje, utilizando metodologías de enseñanza y evaluaciones cualitativas que no solo se utilizan para evaluar al alumno, sino que también buscan evaluar la escuela, su propuesta pedagógica e incluso el sistema educativo.

El aporte de la obra de Paulo Freire, a pesar de ser objeto de múltiples y variadas interpretaciones, ha permitido recrear prácticas educativas en diferentes ámbitos y áreas de actividad en el campo o en la ciudad. En el contexto de las luchas de clases, lo más importante es que los oprimidos tengan una percepción de su condición y busquen la calidad para liberarse de la explotación política y económica. La concepción del conocimiento que recorre la construcción teórica de Freire revela una perspectiva pedagógica progresiva aplicada a la educación, sustentada en una concepción dialéctica, entendida a partir de una articulación compleja de la totalidad de sus dimensiones: política, epistemológica y estética (CARRILLO; OLMOS y CUSATI, 2019).

Es evidente, por lo tanto, la importancia de los estudios que se realicen sobre el paradigma del pensamiento del docente en cuanto a los aportes para conceptualizarlo como profesional, interviniendo en los cursos de formación y en la búsqueda de la profesionalización de la docencia frente a la desvalorización de esta profesión. 


\section{Factores que influyen en el Desarrollo Profesional de los docentes: reinventando la formación}

García (1999, p. 137) se refiere al desarrollo profesional de los docentes como un dispositivo en evolución y continuidad que proporciona la integración de conocimientos relacionados con el currículo, la escuela, la docencia y los docentes. Así, el desarrollo de la escuela está intrínsecamente relacionado con el desarrollo profesional de los docentes y los conocimientos adquiridos en procesos de esa naturaleza.

Para Ponte $(1994 ; 1995 ; 2005)$, un factor preponderante que influye en el desarrollo profesional del docente de matemáticas y el desarrollo de los docentes en otras áreas es el compromiso personal.

El docente debe, en primer lugar, comprometerse a crecer profesionalmente, porque nada es más determinante en el aprendizaje del docente que su propia voluntad de desarrollo. Para ello, se debe desarrollar una postura investigadora, una forma de ser como postulan los investigadores Wenger (1998) e Cochran-Smith \& Lytle (2009). Cochran-Smith \& Lytle (2009) reconocen la importancia del conocimiento generado por los docentes en las comunidades investigadoras y defienden la articulación entre conocimiento académico y conocimiento práctico para la constitución de la profesionalidad docente.

El profesor de matemáticas, necesariamente, necesita estar comprometido e incómodo con las situaciones actuales de su práctica diaria. Sin embargo, deben desarrollar aspectos relacionados con la idea de postura investigadora: concepción del conocimiento local en contextos globales; visión ampliada de la práctica; comunidades de investigación como un medio o mecanismo primario para adoptar una teoría de la acción y la justicia social.

Para Candau (1996), la formación continua de los docentes se configura como un aspecto especialmente crítico y pertinente para la implementación de cualquier propuesta que proponga una renovación de las prácticas pedagógicas y de las escuelas.

La renovación y mejora de la calidad en el desempeño de los docentes y, en consecuencia, en el aprendizaje, implica considerar, según Gatti y Barreto (2009), la formación docente, las perspectivas de carrera y profesionales. García (2009) sostiene que los docentes son la clave para comprender la dinámica del cambio y pensar en los 
escenarios educativos futuros. También advierte de la necesidad de entender "que la profesión docente y su desarrollo son un elemento fundamental y crucial para asegurar la calidad del aprendizaje de los estudiantes" (GARCÍA, 2009, p. 19). "El Desarrollo Profesional busca promover el cambio con los docentes, para que puedan crecer como profesionales - y también como personas" (GARCÍA, 2009, p. 15).

El Desarrollo Profesional de los docentes de matemáticas, según Stein (1998), está atravesado por elementos que dificultan el proceso de cambio de estos docentes e incluso dificultan que surja el deseo de cambios. Entre ellos: la extensa carga de trabajo; el deseo de trabajar de forma aislada; la dificultad de trabajar junto con otros profesores. Todos estos elementos, que en ocasiones minan la motivación del docente para su desarrollo, retraen la autonomía docente. Otro elemento que puede disuadir al docente de iniciar una búsqueda de Desarrollo Profesional está relacionado con las diversas formas de seguimiento externo del estándar docente y la publicación de resultados en pruebas estandarizadas. En estas situaciones, el maestro y la escuela se convierten en rehenes de un sistema sediento de resultados cuantitativos y de los reformadores de políticas de la escuela que ignoran las condiciones reales de los estudiantes. Sin embargo, cuando el deseo de cambio profesional es imperativo, muchos de estos elementos que podrían ser desmotivadores para el desarrollo de los docentes pueden, si no desaparecer, tener su influencia debilitada (GARCÍA, 2009).

En cuanto a los aspectos que pueden estimular el Desarrollo Profesional, se hace hincapié en la búsqueda de una identidad profesional, que es la forma en que "los docentes se definen a sí mismos y a los demás" (GARCÍA, 2009, p. 5). Identidad profesional traduce la interpretación que los docentes tienen de sí mismos en el contexto en el que viven y la importancia que cada uno asigna a su imagen en relación con la profesión, marcada por un sentido de pertenencia a lo largo de la carrera docente, que puede ser individual o colectiva siempre que el docente esté En otras palabras, García (2009) refuerza que el docente es un sujeto social insertado en un entorno escolar y fuera de él, en su vida personal, lo que influye en la formación de su identidad al tener creencias, deseos, certezas y convicciones sustentadas en aspectos sociales y conceptuales como la cultura, religión, antecedentes familiares, conocimientos sobre las materias que impartes en el aula, experiencia en el aula y todo esto puede influir la forma en que se desarrollará. 
Durante las últimas décadas, según Zeichner (2008), la discusión en torno a las ideas del docente reflexivo se centró en los procesos de toma de decisiones por parte de los docentes involucrados en el proceso de enseñanza encaminados a identificar y resolver rápidamente problemas inmediatos. Este problema de investigación, sin embargo, no es actual porque John Dewey, a mediados del siglo XX, ya enfatizó sobre el ejercicio de la acción reflexiva por parte de los profesores. Más allá de lo que actualmente se llama epistemología de la práctica reflexiva, Dewey (1953; 1959) afirma la necesidad de educar al pensamiento para superar su forma rudimentaria hacia el pensamiento reflexivo. Sin embargo, el desarrollo de la práctica reflexiva solo puede ocurrir en base a la necesidad de resolver un problema, que puede surgir a través de la experiencia.

Es en este sentido que la acción reflexiva, propuesta por Dewey $(1953,1959)$, aplicada en la formación inicial y continua del profesorado se presenta como una posibilidad de romper con los modelos estandarizados para convertirse en un punto de partida hacia una racionalidad práctica. Para ello, las experiencias prácticas desde los primeros años de formación deben orientar los programas, teniendo por base la investigación reflexiva en la práctica pedagógica.

Las palabras de Dewey (1959, p. 169) son sustanciales: “[...] la pregunta más importante que se puede hacer sobre cualquier situación o experiencia que tenga el propósito de hacernos aprender algo, es la calidad los problemas involucrados en ellos".

Años más tarde, en 1983, Donald Schön publicó el libro titulado 'El profesional reflexivo' que se convirtió en un referente sobre el tema. En este libro, el autor considera algunos tipos de reflexión que, en el caso específico del maestro de matemáticas, es plausible considerar por tener relación con lo que Schön (1998, p. 29) denominó talento artístico profesional, que son "tipos de competencias que los profesionales demostrar en determinadas situaciones de la práctica que son únicas, inciertas y conflictivas”. ¿Por qué, entonces, considerar estos aspectos señalados por Schön en relación al profesor de matemáticas? Porque es común encontrar testimonios de profesores que reportan malestar ante la desmotivación de los estudiantes.

Según Ponte (1994; 1995), suelen ocurrir dos cosas para resolver este conflicto: la acomodación, en la que el docente decide por la solución más simple como, por ejemplo, impartir una clase preparada e ignorar la reacción de los alumnos, o reflexionar 
sobre la situación conflictiva y cambiar la ruta de navegación como alternativa para llamar la atención de los estudiantes. Esta toma de decisiones, que es rápida, dado el contexto, parece estar subvencionada por el conocimiento del profesor sobre posibles alternativas a la situación, aunque todavía no las haya puesto en práctica previamente.

Según Zeichner (2008), el movimiento en defensa de una práctica reflexiva del profesor o enseñanza reflexiva surgió como una reacción al paradigma vigente en ese momento, en el que se veía a los docentes como técnicos que "meramente hacen lo que otras personas, fuera de en el aula, quieren que lo hagan [...]", incluidos "solo como participantes pasivos" (ZEICHNER, 2008, p. 5). En contraste con las ideas anteriores, el concepto de maestro reflexivo sostiene que este profesional debe asumir un rol activo en la formulación de los propósitos y propósitos de su trabajo y también asumir roles de liderazgo en las reformas escolares. "Reflexión”, sin embargo, significa que la producción de nuevos conocimientos sobre la docencia no es un rol exclusivo de las universidades y comprende el reconocimiento de que los docentes también tienen teorías que pueden contribuir al desarrollo de un conocimiento básico común sobre buenas prácticas docentes (ZEICHNER, 2008, p. 5).

Muchas veces el docente quiere hacer una formación continua y lo hace, incluso durante el período de la pandemia han surgido muchas posibilidades, pero en las escuelas públicas donde enseñan, estas habilidades no son valoradas y consideradas para su progresión profesional. Sin embargo, es primordial destacar que el pensamiento reflexivo es algo inherente al ser humano y el docente, por la propia naturaleza de su profesión, necesita reflexionar sobre sus acciones. Zeichner (2008) sostiene que la mejora de la práctica profesional del docente se da, inicialmente, a partir de estas acciones, de su práctica diaria, y desconoce que la práctica reflexiva sea efectiva a partir de las experiencias de otros, es decir,

[...] El proceso de comprensión y mejora de la propia enseñanza debe comenzar por reflexionar sobre tu propia experiencia y que el tipo de conocimiento que proviene únicamente de la experiencia de otras personas es insuficiente (ZEICHNER, 2008, p. 539).

Para el autor, la práctica reflexiva ocurre cuando el docente participa de las acciones docentes en su totalidad, desde la elaboración, pasando por la aplicación de esta práctica hasta la evaluación de las mismas, ya que caminar o no cambiar la práctica en el aula puede 
ser considerado como un medio que puede potenciar el Desarrollo Profesional del docente, tanto en Matemáticas como en otros docentes (PEREIRA, 2012; PONTE, 2005).

Finalmente, cabe señalar que la efectividad de nuevas metodologías o reformulaciones didácticas, derivadas de acciones reflexivas, puede o no mostrar un cambio de actitud, siempre que el docente tenga un compromiso personal que le ayude a cambiar (PEREIRA, 2012; GARCÍA, 2009; PONTE, 1994; 2005).

Por lo tanto, el desarrollo profesional puede catalizarse si existe compromiso ético y calidad en la práctica profesional, integridad de la práctica docente, responsabilidad individual y comunitaria y compromiso político.

\section{Contribuciones de la educación matemática en la formación y el profesionalismo docente}

El concepto de profesionalismo docente aparece relacionado con la calidad de la práctica profesional, la integridad de la práctica docente y el desarrollo profesional, con el desempeño, valores e intenciones que rigen el proceso docente y con los objetivos que se pretende alcanzar y desarrollar en el ejercicio de la profesión (GARCÍA, 2009; CONTRERAS, 2012). Al traducir la forma en que el docente concibe y vive la labor docente, definió tres dimensiones de la profesionalidad: obligación moral, compromiso con la comunidad y competencia profesional. Para Contreras (2012), la profesión docente no puede analizarse fuera de las dimensiones sociopolíticas, ya que se estima la educación escolar por su importancia cultural y social.

La investigación bibliográfica y documental realizada sobre la formación del profesorado (PEREIRA, 2012) del profesor de matemáticas, concluyó en la necesidad de cualificación, formación, rescate de la profesionalidad y las políticas de profesionalización docente ante la devaluación y precariedad de la formación para la profesión docente. Educación básica.

Ponte (2005) y Gatti et. al. (2011, 2019) muestran que los planes de estudio de la licenciatura en matemáticas no articulan conocimientos técnico-científicos y pedagógicos, desvelando la dicotomía entre matemáticas académicas y escolares.

García (1999) sostiene que la formación docente es un área de conocimiento, investigación, propuestas teóricas y prácticas, que en el ámbito de la didáctica y la 
organización escolar permite el desarrollo de la docencia, el currículo y la escuela, con el objetivo de mejorar educación de los estudiantes.

Los docentes, en su proceso de aprendizaje profesional, pasan por diferentes etapas y momentos impregnados por la complejidad del acto de enseñar. La devaluación de la teoría y el culto a la práctica ha derivado, en las matemáticas, en un movimiento pendular que produjo en la formación inicial de los docentes una excesiva inclinación hacia lo que se consideraba como "práctica".

El aporte de las instituciones de formación inicial a la reinvención de la profesión docente parte del reconocimiento de la importancia y rupturas frente a idiosincrasias curriculares y pedagógicas que vinculan las experiencias escolares de los futuros docentes con los supuestos conceptuales de un paradigma pedagógico instruccional.

Según Trindade (2020, p. 298), "el desajuste entre las exigencias de la vida en lo que hoy se llama sociedades del conocimiento y las propuestas educativas que se construyen bajo la égida del paradigma de la instrucción" confrontan los supuestos, propósitos, desafíos, demandas y necesidades educativas del mundo contemporáneo. Para profundizar en esa reflexión, la investigadora propone la reinvención de la profesión docente

quer como resposta àqueles desafios, exigências e necessidades quer como opção a equacionar, entre outras iniciativas a empreender, face ao mal-estar pessoal e profissional dos professores e à necessidade destes encontrarem novos sentidos e significados para a profissão que abraçaram (TRINDADE, 2020, p. 297).

¿Cuál es la alternativa que puede contribuir a la reinvención de la profesión docente? Trindade (2020) sostiene que es el modelo del docente como interlocutor calificado, y no el modelo del docente como facilitador, lo que debe servir de referencia para una redefinición del trabajo docente que debe pasar por la educación superior. Las razones que sustentan tal enfoque se justifican por las implicaciones inmediatas en la reconfiguración de los proyectos iniciales de formación docente y en un proceso de construcción de un ethos profesional.

La titulación en las matemáticas se basa en estudios históricos, educativos y/o socioculturales de diferentes pueblos y el uso de la interculturalidad como fundamento y movilizador para el desarrollo de las investigaciones en el aula asegura algunos elementos esenciales de una sólida educación matemática. Su importancia en los círculos de educación matemática es fáctica en la medida en que lleva em cuente valor real a los 
aspectos culturales de las sociedades humanas y su potencial pedagógico surge como una propuesta metodológica que resalta las diferencias en el espacio y en el momento, resaltando la relación existente entre cultura, matemática y la educación.

Es una perspectiva poderosa, que sugiere la necesidad de un cuestionamiento y renovación permanente, capaz de corregir fallas y enfrentar los nuevos desafíos que surgen en el camino de la investigación y estudio de las realidades de la práctica (GARCIA BLANCO, 2005).

$\mathrm{Al}$ enfocarse en lo que significa ser docente hoy y enfrentar los obstáculos de la construcción histórico-político-social de la profesión docente, se identifican múltiples factores en la constitución de la formación docente y en la representación que el docente tiene de su profesión en este momento.

Así, la formación de un docente de matemáticas con una postura investigadora, crítica y ética se concibe como una condición previa para la práctica profesional que, bajo las exigencias de plenitud y praxis, busca romper la idea de teoría/práctica/historia como dimensiones estandarizadas. en su formación y que también puede sustentar el surgimiento de una acción profesional vinculada a la defensa de los derechos sociales en la garantía de la ciudadanía y la democracia.

\section{Consideraciones finales}

El clima de renovación educativa de los años de 1930 y la percepción de los pioneros en relación a los problemas educativos y sociales de la época se adelantaron a ese período. La transformación social requerida y muchos problemas señalados por los signatarios en 1932, en relación con la educación, se encuentran aún hoy, independientemente del posicionamiento político e ideológico del Manifiesto, lo que indica su relevancia histórica.

De todas formas, se refuerza la afirmación de que habrá un cambio en la formación de los futuros profesores de matemáticas, aspirando a que la universidad, la escuela y la comunidad estén más cerca, brindando experiencias de aprendizaje ampliadas que se crean en el ínterin del juego interactivo de diferentes fuentes de conocimiento. También es relevante fomentar el contacto con el entorno y el establecimiento del dialogo intercultural en un ambiente de respeto a la diversidad, así 
como impulsar una educación pertinente con la cultura del entorno inmediato de los estudiantes, que incorpore elementos y contenido de horizontes culturales diversos.

A pesar de la complejidad de asumir, en las instituciones de formación, esta nueva epistemología de la formación docente, se recomienda un vínculo con la concepción ontológica del ser social y la construcción de un ethos profesional crítico, con bases reflexivas sobre la realidad social y la responsabilidad de los Intervención profesional ética en esta realidad.

Los aportes presentados por los académicos son fundamentales para orientar el desempeño formativo y docente en cuanto a la movilización y construcción del conocimiento necesario para la docencia, es decir, la profesionalización de la docencia. Esta profesionalización, que involucra una dimensión epistemológica (naturaleza del conocimiento involucrado) y también una dimensión política, puede subsidiar la construcción de la identidad profesional del profesorado, indispensable para el estatus de profesionalización docente.

Para la comprensión de la profesión docente, necesita tener en cuenta los valores, las normas, los discursos, los principios que interiorizan los sujetos a lo largo de sus trayectorias de vida y, también, la forma en que los exteriorizan, transformándolos en actos. de la realidad objetiva. Junto a los valores y principios, también es necesario considerar las politizaciones subjetivas, los medios de producción y las ganancias de poder que componen la labor docente y la formación de prescripciones.

A partir del acceso a estos aspectos relacionados con la labor docente, quizás se puedan comprender las elecciones y actitudes que caracterizan alguna subjetividad posible de cada docente, sus definiciones en relación a la concepción de alumno, educación, escuela y su propio rol social.

Al final, cabe señalar que la formación continua es fundamental en la vida profesional de los docentes para que se especialicen y actualicen de cara al nuevo escenario educativo.

\section{Referencias}

AVALOS, B. Los conocimientos y las competencias que subyacen a la tarea docente. In: MEDRANO, C. V.; VAILLANT, D. (Coord.). Aprendizaje y desarrollo profesional docente. Madrid, Espanha: Fundación Santillana, 2021. p. 67-77. (Colección Metas Educativas 2021). 
BICUDO, M. A. V. Filosofia da educação matemática: sua importância na formação de professores de matemática. São Paulo: Moraes, 1987.

BISHOP, A. J. Enculturación matemática: la educación matemática desde una perspectiva cultural. Barcelona: Paidós, 1991.

BRASIL. LDB: Lei de Diretrizes e Bases da Educaşão Nacional: lei 9.394 de 20 de dezembro de 1996, que estabelece as diretrizes e bases da educação nacional. 5.ed. Brasília: Câmara dos Deputados, Coordenação Edições Câmara, 2010.

BRASIL. Ministério da Educação. Plano Nacional da Educação. Brasília, DF, 2014.

CANDAU, V. M. Formação continuada de professores: tendências atuais. In: REALI, A. M. M. R. et al. Formação de professores: tendências atuais. São Carlos: EDUFSCAR, 1996. p. 139-165.

CARRILLO, A.; OLMOS, A.; CUSATI, I. C. Articulações entre educação intercultural e práticas de internacionalização entre o México e o Brasil. RIAEE - Revista Ibero-Americana de Estudos em Educação, Araraquara, v. 14, n. 3, p. 1182-1204, jul./set., 2019.

COCHRAN-SMITH, M.; LYTLE, S. Inquiry as stance: practitioner research for the next generation. New York: Teachers College Press, 2009.

CONTRERAS, J. A autonomia de professores. Trad. Sandra Trabucco Venezuela. 2. ed. São Paulo: Cortez, 2012.

CUNHA, M. I. Narrativas e formação de professores: uma abordagem emancipatória. In: SOUZA, E. C.; GALLEGO, R. C. (org.). Espaços, tempos e gerações: perspectivas (auto) biográficas. São Paulo: Cultura Acadêmica, 2010. p. 199-214.

DEWEY, J. Como Pensamos. São Paulo: Companhia Editora Nacional, 1953.

DEWEY, J. Democracia e Educação. São Paulo: Companhia Editora Nacional, 1959.

DINIZ-PEREIRA, J. E. A prática como componente curricular na formação de professores. Educação (UFSM), Santa Maria, v. 36, n. 2, p. 203-218, maio/ago. 2011. Disponível em: https://periodicos.ufsm.br/reveducacao/article/view/3184. Acesso em: 26 de jun. 2020.

FREIRE, P. Pedagogia dos sonhos possíveis. São Paulo: UNESP, 2001.

GARCIA BLANCO, M. M. La formación de profesores de matemáticas: Un campo de estudio y preocupación. Educación Matemática, Santillana, v. 17, n. 2, p. 153-166, 2005.

GARCÍA, C. M. Desenvolvimento profissional docente: passado e futuro. Sísifo - Revista de Ciência da Educação, n. 8, p. 7-22, jan./abr. 2009. Disponível em:

http://www.unitau.br/files/arquivos/category_1/MARCELO__Desenvolvimento_Profi ssional_Docente_passado_e_futuro_1386180263.pdf. Acesso em: 26 de jun. 2020.

GARCÍA, C. M. Formação de professores. Para uma mudança educativa. Trad. Isabel Narciso. Porto/PORTUGAL: Porto Editora, 1999.

GATTI, B. A. et al. Professores do Brasil: novos cenários de formação. Brasília: UNESCO, 2019. 
GATTI, B. A.; BARRETO, E. S. S. Professores do Brasil: impasses e desafios. Brasília: UNESCO, 2009.

GATTI, B. A.; BARRETO, E. S. S.; ANDRÉ, M. E. D. A. Politicas docentes no Brasil: um estado da arte. Brasília: UNESCO, 2011.

KLEIN, F. Matemática Elementar de um Ponto de Vista Superior. Volume I, Parte I: Aritmética. Lisboa: Sociedade Portuguesa de Matemática, 2009.

LIMA, M. S.; PIMENTA, S. G. Estágio e Docência: diferentes concepções. In: LIMA, M. S.; PIMENTA, S. G. Estágio e Docência. São Paulo: Cortez, 2004.

MIZUKAMI, M. G. N. Aprendizagem da docência: algumas contribuições de L. S. Shulman. Revista do Centro de Educação, Santa Maria, v. 29, n. 2, p. 33-49, 2004.

MIZUKAMI, M. G. N. Aprendizagem da docência: professores formadores. Revista E-Curriculum, São Paulo, v. 1, n. 1, dez./jul. 2005-2006. Disponível em: http://www.pucsp.br/ecurriculum. Acesso em: 21 de jun. de 2020.

MOTEJUNAS, P. R. A evolução do ensino da Matemática no Brasil. p. 150-163. In: GARCIA, E. W. (coord.). Inovação Educacional no Brasil: Problemas e Perspectivas. São Paulo: Cortez Autores Associados, 1989.

NÓVOA, A. Para uma formação de professores construída dentro da profissão. In: NÓVOA, A. Professores: Imagens do futuro presente. Lisboa: Educa, 2009. p. 25.46

PEREIRA, M. V. Nos supostos para pensar formação e autoformação: a professoralidade produzida no caminho da subjetivação. In: FRAZÃO, C. et al. Ensinar e aprender: sujeitos, saberes e pesquisa. 2. ed. Rio de Janeiro: DP\&A, 2012, p. 23-41.

PIMENTA, S. G. (org.). Saberes pedagógicos e atividade docente. 2. ed. São Paulo: Cortez, 2000.

PIMENTA, S. G.; GONÇALVES, C. L. Revendo o ensino de $2^{\circ}$ grau: propondo a formação de professores. 2. ed. rev. São Paulo: Cortez, 1992.

PONTE, J. P. O Desenvolvimento Profissional do professor de Matemática. Educação e Matemática, n. 31, p. 9-20, 1994. Disponível em:

http://repositorio.ul.pt/bitstream/10451/4474/1/94\%20Ponte\%20EM31\%20pp0912_20. pdf. Acesso em: 28 jun. 2020.

PONTE, J. P. Perspectivas de desenvolvimento profissional de professores de Matemática. In: PONTE, J. P. et al. (Eds.). Desenvolvimento profissional de professores de Matemática: Que formação? Lisboa: SEM-SPCE, 1995. p. 193-211.

PONTE, J. P. A vertente profissional da formação inicial de professores de Matemática. Educação Matemática em Revista - SBEM, ano 9, n. 11, p. 3-8, 2002. Disponível em: http://www.educ.fc.ul.pt/docentes/jponte/docs-pt/02-Ponte\%20(SBEM).pdf. Acesso em: 27 jun. 2020.

PONTE, J. P. A formação do professor de Matemática: Passado, presente e futuro. In: SANTOS, A. P. C.; BROCARDO, J. (Eds.). Educaşão matemática: Caminhos e encruzilhadas. Lisboa: APM, 2005, p. 267-284. Disponível em: 
http://repositorio.ul.pt/handle/10451/3169. Acesso em: 09 jun. 2020.

RANGEL, L.; GIRALDO, V.; MACULAN, N. Matemática elementar e saber pedagógico de conteúdo — estabelecendo relações. In: COLÓQUIO DE HISTÓRIA E TECNOLOGIA NO ENSINO DE MATEMÁTICA (VI HTEM), 6., 2013, São Carlos. Anais... São Carlos, SP: UFSCar, 2013.

SCHÖN, D. El profesional reflexivo. Como piensan los profesionales cuando actúan. Paidós: Barcelona, 1998.

STEIN, M. K.; SILVER, E. A. e SMITH, M. S. Mathematics reform and teacher development: a Community of practice perspective. In: GREENO, J. e GOLDMAN, S. (Orgs.). Thinking practices in mathematics and Science learning. Hildale, Laurence Erlbaum Ass., 1998. p. 17-52.

TEIXEIRA, A. O problema da formação do magistério. Rev. Bras. Est. Ped., Rio de Janeiro, v. 46, n. 104, p. 278-297, 1996. Disponível em:

http://rbepold.inep.gov.br/index.php/rbep/article/view/924/899. Acesso em: 13 jun. 2020.

TRINDADE, R. A formação de professores e o seu contributo para a reinvenção da profissão docente. Revista Cocar. Edição Especial, n. 8, p. 293-314, jan./abr. 2020.

Disponível em: https://paginas.uepa.br/seer/index.php/cocar/index. Acesso em: 10 jun. 2020.

WENGER, E. Communities of practice: learning, meaning, and identity. Cambridge: Cambridge University Press, 1998.

ZEICHNER, K. M. Formação de professores para a justiça social em tempos de incerteza e desigualdades crescentes. In: DINIZ-PEREIRA, J. E.; ZEICHNER, K. M. (Orgs.). Justiça Social: desafio para a formação de professores. Belo Horizonte: Autêntica Editora, 2008.

ZEICHNER, K. M. Repensando as conexões entre a formação na universidade e as experiências de campo na formação de professores em faculdades e universidades. Educação (UFSM), Santa Maria, v. 35, n. 3, p. 479-504, set./dez. 2010. Disponível em: https://periodicos.ufsm.br/reveducacao/article/view/2357. Acesso em: 26 jun. 2020.

ZEICHNER, K. M. Políticas de formação de professores nos Estados Unidos: como e porque elas afetam vários países no mundo. Belo Horizonte: Autêntica Editora, 2013.

RECIBIDO: 30/11/2020

APROBADO: 28/01/2021
RECEIVED: 30/11/2020

APPROVED: 01/28/2021
RECEBIDO: 30/11/2020

APROVADO: 28/01/2021 